\title{
Evaluación de las competencias del docente bajo la percepción de los estudiantes de la escuela profesional de administración de la Universidad Nacional Santiago Antúnez de Mayolo, 2016
}

Evaluation of the competences of the teacher under the perception of the students of the professional school of administration of the National University Santiago Antúnez de Mayolo, 2016

\author{
Guillermo Peláez díaz ${ }^{1}$, Juan Vílchez Cárcamo ${ }^{1}$, Lorenzo Valderrama plasencia ${ }^{1}$ y Milady \\ Carbajulca Milla ${ }^{1}$
}

\section{RESUMEN}

Determinar la percepción que tienen los alumnos sobre las competencias del docente de la Escuela de Administración de la Universidad Nacional Santiago Antúnez de Mayolo, según los factores de planificación, interacción didáctica del proceso de enseñanza aprendizaje y valoración del proceso de enseñanza aprendizaje. El diseño de la investigación es no experimental, transversal y descriptivo, la muestra equivale a 219 alumnos de la escuela de Administración del semestre 2016-II, seleccionados mediante el muestreo aleatorio simple. Los resultados del presente estudio muestran que los alumnos en un 57\% han percibido las competencias de los docentes a nivel general como bueno, lo que significa una aproximación significativa del proceso de enseñanza/aprendizaje de la escuela profesional de Administración, de acuerdo a las exigencias de la Superintendencia Nacional de Educación Superior Universitaria, en el marco del sistema de aseguramiento de la calidad y en aras de lograr el licenciamiento en la escuela profesional de Administración.

Palabras clave: evaluación; competencias del docente; percepción de los estudiantes.

\section{ABSTRACT}

Decide the students' perception of the competences of the teacher of the School of Administration of the National University Santiago Antunez de Mayolo, according to the planning factors, didactic interaction of the teaching learning pro-

1 Universidad Nacional Santiago Antúnez de Mayolo. Huaraz, Perú. 
cess and evaluation of the process of teaching learning. The research design is non-experimental, transversal and descriptive, the sample is equivalent to 219 students of the administration school of the semester 2016-II, selected through simple random sampling. The results of the present study show that students in $57 \%$ have perceived the competences of teachers at the general level as good, which means a significant approximation of the teaching / learning process of the professional school of Administration, according to the Requirements of the National Superintendence of Higher University Education, within the framework of the quality assurance system and in order to achieve the licensing in the professional school of Administration.

Keywords: evaluation; teacher competencies; student perceptions.

\section{INTRODUCCIÓN}

Según un estudio realizado por López, Benedito y León (2016), los resultados obtenidos evidencian que la incorporación del enfoque de competencias a la formación universitaria representa una oportunidad para ensalzar el valor formativo de la práctica. Sin embargo, su adopción puede suponer una vuelta a posicionamientos tecnológicos ampliamente cuestionados y una subordinación a las demandas del mercado. Para evitarlo, el panel de expertos propone mejorar las condiciones en las que se ejerce la enseñanza, prestar mayor atención a la formación del profesorado, y ampliar la reflexión y debate en torno al enfoque de competencias y a los sistemas de evaluación. De otro lado, conviene mencionar el estudio de Haskell (1997), citado por García (2000), este autor, basado en una extensa revisión bibliográfica, advierte que el uso de los cuestionarios de evaluación con propósitos sumativos atenta contra la libertad académica y el derecho a la definitividad en el empleo de los profesores; por esas razones, Haskell concluye que los cuestionarios de evaluación no son tan válidos.

Así también, en la evaluación de competencias pedagógicas, cabe destacar el estudio realizado por Caballero y Sime (2016), quienes indican que "buen o buena docente" es aquella persona que ha marcado e influido de manera trascendental en la vida personal y académica de sus estudiantes y cuya imagen permanece en su memoria como un recuerdo prolongado y altamente positivo. En consecuencia, conocer cuáles son sus rasgos centrales y por qué impactaron de tal manera en sus estudiantes merece ser más investigado como un aporte al campo de estudio sobre la docencia en educación básica. Los resultados de este estudio cualitativo, ha permitido describir, por un lado, los rasgos del buen personal docente en relación a su alumnado, caracterizado por una percepción positiva y actitud motivadora y una calidad afectiva en la interacción profesorado-alumnado; y, por otro lado, rasgos en relación con su profesión como: su pasión por enseñar; su dominio sobre contenidos y la organización de la clase. 
De acuerdo a la Ley Universitaria No 30220 (2014), mediante el artículo $\mathrm{N}^{\circ} 12$ se crea la Superintendencia Nacional de Educación Superior Universitaria (SUNEDU), como Organismo Público Técnico Especializado adscrito al Ministerio de Educación, con autonomía técnica, funcional, económica, presupuestal y administrativa, para el ejercicio de sus funciones y es responsable del licenciamiento para el servicio educativo superior universitario, entendiéndose el licenciamiento como el procedimiento que tiene como objetivo verificar el cumplimiento de condiciones básicas de calidad para ofrecer el servicio educativo superior universitario. Precisamente bajo este marco legal, es que nos hemos trazado como objetivo general: Describir la percepción que tienen los alumnos sobre las competencias del docente en la Escuela de Administración de la Universidad Nacional Santiago Antúnez de Mayolo.

Así mismo, cabe precisar que Odar (2007), manifiesta que las universidades tienen entre sus fines la formación de profesionales de alta calidad. Esta calidad está ligada al servicio que brindan sus profesores, y para mejorar este servicio docente es necesario conocerlo con el mayor detalle posible, para determinar sus virtudes con la finalidad de mejorar, lo que implica la necesidad de evaluar. Evaluar la competencia docente universitaria, desde la perspectiva del alumno es un tema de permanente actualidad, ampliamente debatido, prolijo y complejo que se viene dando a nivel internacional, regional y local.

De acuerdo al Centro interuniversitario de desarrollo CINDA, MINEDUC CHILE (2007), en los cuestionarios que se usan para las encuestas a los estudiantes, se pregunta sobre la percepción del estudiante acerca del dominio que tiene el profesor de los contenidos del curso y acerca de su desempeño en la organización y planificación del curso, en la comunicación con los estudiantes, en el manejo de recursos para facilitar el aprendizaje y autoaprendizaje de los estudiantes, y en las evaluaciones de los aprendizajes.

Conviene indicar que el enfoque de competencias promovido desde las esferas oficiales del gobierno ha sido poco persistente, al haberse puesto en marcha un importante proyecto de reforma y no contar con un sólido programa de formación continua de docentes de acuerdo con el actual enfoque. En tal sentido cabe precisar que en la Universidad Nacional Santiago Antúnez de Mayolo se evalúa al docente en cada ciclo académico, aplicando la encuesta estudiantil antes de las pruebas parciales. La encuesta mide aspectos tales como puntualidad en la entrega de sílabos, cumplimiento de objetivos de estudio, etc. sus resultados muestran una valoración que califica al profesor como sobresaliente, muy bueno, bueno, regular y malo.

Según Villa y Villa (2007), se define la competencia como el buen desempeño en contextos diversos y auténticos basada en la integración y la activación de conocimientos, normas, técnicas, procedimientos, habilidades y destrezas, actitudes y valores. Este concepto queda gráficamente expresado en la figura 1: 


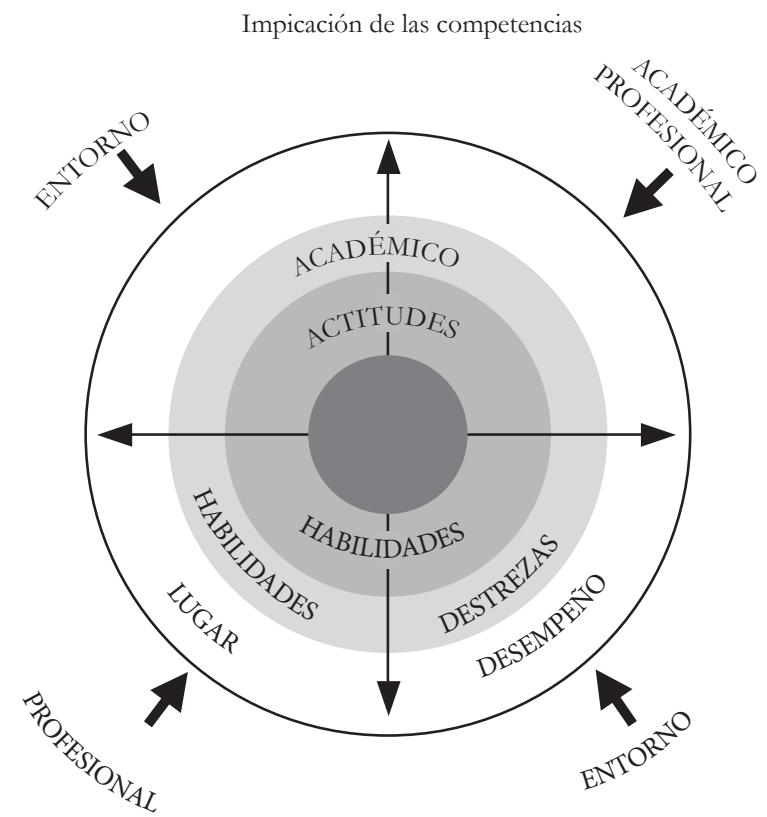

Figura 1. El concepto de competencia en la UD (Poblete, 2003)

Así también Mas (2012), indica que las competencias profesionales son mucho más que una simple acumulación de contenidos (saber), estando también constituidas por habilidades (saber hacer), actitudes y valores (saber ser y estar). Así mismo Echeverría (2002) indica que para el desempeño eficaz de una profesión es necesario saber los conocimientos requeridos, un ejercicio eficaz de estos necesita un saber hacer, saber ser y saber estar. En el caso concreto de la docencia universitaria, el saber se corresponde con las competencias científicas, el saber hacer con competencias metodológicas/técnicas, el saber ser con competencias personales y el saber estar con competencias sociales/participativas.

De igual manera, Smith y Cranton (1992), citados por García (2000), investigaron que las diferencias en las percepciones de los estudiantes sobre las habilidades docentes de 2816 grupos de una facultad de Comercio Canadiense, los autores encontraron que las dimensiones de la efectividad docente varían de acuerdo con el año en que se encuentran cursando los estudios universitarios o si los cursos son de posgrado.

Consecuentemente, el presente estudio se justifica porque determina la percepción que tienen los alumnos sobre las competencias del docente de la Escuela de Administración de la Universidad Nacional Santiago Antúnez de Mayolo, según los factores de planificación, interacción didáctica del proceso de enseñanza aprendizaje y valoración del proceso de enseñanza aprendizaje, utilizando para ello el método hipotético-deductivo, hermenéutico y descriptivo. La sección de Resultados aborda aspectos relacionados con la percepción de los alumnos 
referente a las competencias de los docentes que nos darán una aproximación significativa del proceso de enseñanza/aprendizaje de la escuela profesional de Administración.

\section{MATERIALES Y MÉTODOS}

El método que se utiliza según el enfoque investigativo es cuantitativo, según su alcance es descriptivo. El tipo de investigación es aplicada porque que parte de una situación problemática que requiere ser intervenida y mejorada a favor de la comunidad. Se utiliza el diseño de investigación no experimental, transversal en la modalidad descriptivo. También se utiliza la técnica de revisión bibliográfica, debido a que forma parte integral de toda investigación. La población está compuesta por 440 alumnos de la escuela de Administración, matriculados en el semestre académico 2016-II, de los cuales aleatoriamente se ha extraído una muestra de 219 alumnos, correspondiendo a 20 alumnos por ciclo.

Cabe mencionar que el cuestionario para evaluar la competencia docente por los alumnos, ha sido validado por Luna, Calderón, Caso y Cordero (2012), en su estudio "Diseño, desarrollo y validación de un cuestionario de evaluación de la competencia docente por los estudiantes", amparándose en los factores planificación, interacción didáctica del proceso de enseñanza aprendizaje y valoración del proceso de enseñanza aprendizaje.

\section{RESULTADOS}

La recolección de datos a partir de las encuestas, nos generó información importante que responden directamente a los objetivos de la investigación, en ese sentido se muestran los siguientes resultados:

a) Describir la percepción que tienen los alumnos sobre las competencias del docente en la Escuela de Administración de la Universidad Nacional Santiago Antúnez de Mayolo, 2016:

Tabla 1. Percepción de alumnos de las competencias docentes FAT 2016

\begin{tabular}{ccc}
\hline Escala & $\mathrm{n}$ & $\%$ \\
\hline Malo & 16 & 7.3 \\
Regular & 78 & 35.6 \\
Bueno & 104 & 47.5 \\
Muy bueno & 21 & 9.6 \\
& 219 & 100 \\
\hline
\end{tabular}


Los resultados indican que el $7.3 \%$ de los alumnos perciben las competencias docentes como malas, el $35.6 \%$ de los alumnos lo perciben como regulares, el $47.5 \%$ y el $9.6 \%$ como buenas y muy buenas respectivamente.

b) Describir la percepción que tienen los alumnos sobre las competencias del docente en la planificación del proceso de enseñanza-aprendizaje en la Escuela de Administración de la Universidad Nacional Santiago Antúnez de Mayolo, 2016:

Tabla 2. Percepción de alumnos de la competencia planificación de la enseñanza aprendizaje

\begin{tabular}{ccc}
\hline \multicolumn{3}{c}{ Percepción planificación E-A } \\
\hline & $\mathrm{n}$ & $\%$ \\
Malo & 21 & 9.58 \\
Regular & 83 & 37.9 \\
Bueno & 96 & 43.8 \\
Muy bueno & 19 & 8.7 \\
\hline
\end{tabular}

Los resultados nos indican que el $8.7 \%$ de los alumnos percibe como muy bueno la planificación del proceso E-A; el $43.8 \%$ lo percibe como bueno; un 37.9\% de los alumnos lo percibe como regular y el $9.58 \%$ lo percibe como malo.

c) Describir la percepción que tienen los alumnos sobre las competencias del docente en la interacción didáctica del proceso de enseñanza- aprendizaje en la Escuela de Administración de la Universidad Nacional Santiago Antúnez de Mayolo, 2016:

Tabla 3. Percepción de alumnos de la competencia interacción didáctica del proceso de la enseñanza aprendizaje

\begin{tabular}{ccc}
\hline \multicolumn{3}{c}{ Percepción sobre el proceso de enseñanza aprendizaje } \\
\hline & $\mathrm{n}$ & $\%$ \\
Malo & 25 & 11 \\
Regular & 82 & 34 \\
Bueno & 94 & 43 \\
Muy bueno & 18 & 8.2 \\
\hline
\end{tabular}

Los resultados indican que el 11\% de los alumnos tienen una percepción mala del proceso enseñanza aprendizaje que efectúan los docentes, el $34 \%$ de ellos califican como regular, el $43 \%$ lo califica como buena y el $8.2 \%$ como muy buena. 
d) Describir la percepción que tienen los alumnos sobre las competencias del docente en la valoración del proceso de enseñanza-aprendizaje en la Escuela de Administración de la Universidad Nacional Santiago Antúnez, de Mayolo, 2016:

Tabla 4. Percepción de los alumnos de la valoración del proceso de enseñanza aprendizaje

Percepción de la valoración proceso E-A

\begin{tabular}{ccc}
\multicolumn{3}{c}{$\mathrm{n} \%$} \\
Malo & 15 & 6.8 \\
Regular & 73 & 33.3 \\
Bueno & 105 & 48 \\
Muy bueno & 26 & 11.8 \\
\hline
\end{tabular}

Los resultados nos indican que el 6.8\% de los alumnos perciben la valoración del proceso de enseñanza aprendizaje como malo, el $33.3 \%$ de los alumnos lo percibe como regular; un 48\% lo percibe como bueno y un $11.8 \%$ lo percibe como muy bueno.

e) Contrastación de Hipótesis:

Tabla 5. Contrastación de hipótesis usando la tabla de contingencia y la prueba chi cuadrado

Tabla de contingencia

\begin{tabular}{ccccc}
\hline & \multicolumn{4}{c}{ PERCEPCIÓN DE LAS COMPETENCIAS } \\
\hline & Malo & Regular & Bueno & Muy bueno \\
Alumnos $<=5^{\circ}$ ciclo & 8.11 & 39.53 & 52.71 & 10.64 \\
Alumnos $6^{\circ}$ al $10^{\circ}$ ciclo & 7.89 & 38.47 & 51.29 & 10.36 \\
\hline \multicolumn{5}{c}{ Ji cuadrada $=0.9012$}
\end{tabular}

\section{DISCUSIÓN}

A continuación se analizan los resultados de la investigación sobre Evaluación de las competencias del docente bajo la percepción de los estudiantes de la escuela profesional de Administración de la Universidad Nacional Santiago Antúnez de Mayolo, 2016, como sigue:

a) Describir la percepción que tienen los alumnos sobre las competencias del docente en la Escuela de Administración de la Universidad Nacional Santiago Antúnez de Mayolo, 2016. 
$\mathrm{Al}$ respecto, los resultados de acuerdo a la tabla 1, muestran que los alumnos de administración durante el año 2016 han percibido las competencias de los docentes a nivel general como bueno, situación explicada por el hecho de que el 57\% de los alumnos percibe las competencias por encima de bueno; por otro lado el $83 \%$ de los alumnos percibe las competencias docentes entre regular y bueno y el $42.9 \%$ lo percibe entre malo y regular; porcentaje este último bastante alto, que estaría indicando que aún falta mejorar las competencias de los docentes de la Escuela de Administración. Así mismo a nivel general el 9.6\% de los alumnos perciben las competencias como muy bueno. Cabe mencionar por lo tanto que de acuerdo a los resultados de los cuestionarios de opinión de los estudiantes, se puede apreciar que una gran mayoría (42.9\% lo percibe entre malo y regular) espera que los docentes mejoren sus competencias en temas que tienen que ver con la capacidad para dominar un área determinada, aprender a tratar con tareas bien definidas y brindar respuestas efectivas para la resolución de problemas en un ámbito profesional concreto, con el fin de lograr un nivel de calidad de la enseñanza universitaria significativo. Todo esto coincide con Odar (2007), quien manifiesta que las universidades tienen entre sus fines la formación de profesionales de alta calidad. Esta calidad está ligada a la calidad de servicio que brindan sus profesores, y para mejorar este servicio docente es necesario conocerla con el mayor detalle posible, para determinar sus virtudes con la finalidad de mejorarla, lo que implica que hay que evaluarla desde la perspectiva del alumno.

b) Describir la percepción que tienen los alumnos sobre las competencias del docente en la planificación del proceso de enseñanza-aprendizaje en la Escuela de Administración de la Universidad Nacional Santiago Antúnez. de Mayolo, 2016.

Sobre el particular, los resultados de acuerdo a la tabla 2, muestran que los alumnos de administración durante el año 2016 en un $8.7 \%$ lo percibe como muy bueno, el $43.8 \%$ como bueno, el $37.9 \%$ como regular y el $9.58 \%$ como malo. Consecuentemente, queda claro que según la opinión de los estudiantes, se puede apreciar un gran porcentaje de ellos $(47.5 \%$ lo percibe entre malo y regular) que espera que los docentes planifiquen adecuadamente el proceso de enseñanza-aprendizaje, en temas que se relacionen con el dominio que debe poseer el profesor de los contenidos del curso, así como también de su desempeño en la organización y en la planificación del curso, todo ello a fin de contribuir progresivamente a la mejora del nivel de calidad de la enseñanza universitaria. Estos resultados ratifican lo señalado por el Centro interuniversitario de desarrollo CINDA, MINEDUC CHILE (2007), que indica que en los cuestionarios que se usan para las encuestas a los estudiantes, se pregunta sobre la percepción del estudiante acerca del dominio que tiene el profesor de los contenidos del curso y acerca de su desempeño en la organización y planificación del curso, 
en la comunicación con los estudiantes, en el manejo de recursos para facilitar el aprendizaje y autoaprendizaje de los estudiantes, y en las evaluaciones de los aprendizajes.

c) Describir la percepción que tienen los alumnos sobre las competencias del docente en la interacción didáctica del proceso de enseñanza-aprendizaje en la Escuela de Administración de la Universidad Nacional Santiago Antúnez de Mayolo, 2016:

Sobre esta competencia, los resultados según la tabla 3, indican que el 11\% de los alumnos tienen una percepción mala de la interacción didáctica del proceso enseñanza aprendizaje que efectúan los docentes, el $34 \%$ de ellos califican como regular, el 43\% lo califica como buena y el 8.2 \% como muy buena. Esto implica que gran porcentaje de los alumnos (45\% lo califican de regular hacia abajo), esperan que los docentes perfeccionen el saber organizar y secuenciar adecuadamente los contenidos del aprendizaje, conocer la metodología y la técnicas de investigación, tener la formación pedagógica necesaria para dirigir el proceso de $\mathrm{E} / \mathrm{A}$, elaborar una guía de la asignatura que resulte útil y práctica para el alumno, ser capaz de preparar, seleccionar y construir actividades y materiales adecuados para el trabajo en el aula, utilizar eficazmente las tutorías, y aplicar estrategias y técnicas que enseñen a los alumnos a trabajar en equipo. Este resultado es concordante con Mas (2012), quien indica que las competencias profesionales son mucho más que una simple acumulación de contenidos (saber), estando también constituidas por habilidades (saber hacer), actitudes y valores (saber ser y estar). También coincide con Villa y Villa (2007), quienes definen la competencia como el buen desempeño en contextos diversos y auténticos basado en la integración y la activación de conocimientos, normas, técnicas, procedimientos, habilidades y destrezas, actitudes y valores que el docente debe poseer.

d) Describir la percepción que tienen los alumnos sobre las competencias del docente en la valoración del proceso de enseñanza-aprendizaje en la Escuela de Administración de la Universidad Nacional Santiago Antúnez de Mayolo, 2016.

Los resultados según la tabla 4 , nos indican que el 6.8\% de los alumnos perciben la valoración del proceso de enseñanza aprendizaje como malo, el 33.3\% de los alumnos lo percibe como regular; un 48\% lo percibe como bueno y un $11.8 \%$ lo percibe como muy bueno. Este resultado incluye un alto porcentaje de alumnos (40.1\% lo aprecian de regular hacia abajo), que esperan que los docentes mejoren sus competencias tales como: ser capaz de reflexionar críticamente sobre la propia práctica docente, para introducir elementos de cambio y mejora, ser correcto y respetuoso en el trato con el alumnado, potenciar en el alumnado actitudes de participación y colaboración como miembros activos de la comunidad educativa, colaborar con otros profesores/as y trabajar en equipo para diseñar programas y asignaturas coherentes y adaptadas, diseñar estrategias 
didácticas y metodológicas que faciliten el proceso de enseñanza-aprendizaje del alumnado, participar en proyectos de innovación educativa que mejoren la calidad de la enseñanza y mantener una imagen personal. Estos resultados están en concordancia con Echeverría (2002), quien indica que para valorar el proceso de enseñanza-aprendizaje, es necesario saber los conocimientos requeridos por la misma, un ejercicio eficaz de estos necesita un saber hacer, saber ser y saber estar. En el caso concreto de la docencia universitaria, el saber se corresponde con las competencias científicas, el saber hacer con competencias metodológicas/técnicas, el saber ser con competencias personales y el saber estar con competencias sociales/participativas.

e) Contrastación de Hipótesis. Con relación a la prueba de contingencia realizada agrupando la valoración de los alumnos que cursan entre el $1^{\circ}$ ciclo al $5^{\circ}$ ciclo y los alumnos que cursan entre el $6^{\circ}$ ciclo al $10^{\circ}$ ciclo; se observa que la prueba Chi cuadrada muestra un valor de 0.91 para un valor teórico de 3.59, indicándonos que existen diferencias en las percepciones que tienen los estudiantes de ambos grupos analizados en la escuela profesional de Administración. Por consiguiente, estos resultados son concordantes con la investigación de Smith y Cranton (2012) citado por García (2000), quienes indican que las diferencias en las percepciones de los estudiantes sobre las habilidades docentes de 2816 grupos de una facultad de Comercio Canadiense, los autores encontraron que las dimensiones de la efectividad docente varían de acuerdo con el año en que se encuentran cursando los estudios universitarios, o si los cursos son de posgrado.

\section{CONCLUSIONES}

Se hace necesario mejorar las competencias de los docentes de la Escuela de Administración frente al $42.9 \%$ de alumnos que lo percibe entre malo y regular, esperando que los docentes mejoren sus competencias en temas que tienen que ver con la capacidad para dominar un área determinada, aprender a tratar con tareas bien definidas y brindar respuestas efectivas para la resolución de problemas en un ámbito profesional concreto, con el fin de lograr un nivel de calidad de la enseñanza universitaria significativo y acorde con en el marco del sistema de aseguramiento de la calidad que nos faculte el licenciamiento en la escuela profesional de Administración.

Frente al $47.5 \%$ de estudiantes que perciben entre malo y regular la previsión o planeamiento del proceso de enseñanza/aprendizaje, se espera que los docentes se superen en temas que se relacionen con el dominio que debe poseer el profesor de los contenidos del curso, así como también de su desempeño en la organización y en la planificación del curso, todo ello a fin de contribuir progresivamente a la mejora del nivel de calidad de la enseñanza universitaria. 
Respecto al 45\% de estudiantes que califican de regular hacia abajo, la interacción didáctica del proceso de enseñanza/aprendizaje, se espera que los docentes perfeccionen el saber organizar y secuenciar adecuadamente los contenidos del aprendizaje, conocer la metodología y la técnicas de investigación, tener la formación pedagógica necesaria para dirigir el proceso de E/A, elaborar una guía de la asignatura que resulte útil y práctica para el alumno, ser capaz de preparar, seleccionar y construir actividades y materiales adecuados para el trabajo en el aula, utilizar eficazmente las tutorías, emplear con eficacia las TICs y aplicar estrategias y técnicas que enseñen a los alumnos a trabajar en equipo.

En relación al 40.1\% de estudiantes que aprecian de regular hacia abajo la valoración del proceso de enseñanza/aprendizaje, se espera que los docentes mejoren sus competencias sociales y participativas tales como: ser capaz de reflexionar críticamente sobre la propia práctica docente, para introducir elementos de cambio y mejora, ser correcto y respetuoso en el trato con el alumnado, potenciar en el alumnado actitudes de participación y colaboración como miembros activos de la comunidad educativa, colaborar con otros profesores/as y trabajar en equipo para diseñar programas y asignaturas coherentes y adaptadas, diseñar estrategias didácticas y metodológicas que faciliten el proceso de enseñanza-aprendizaje del alumnado, participar en proyectos de innovación educativa que mejoren la calidad de la enseñanza y mantener una imagen personal cuidada.

\section{REFERENCIAS BIBLIOGRÁFICAS}

Caballero, Robert y Sime, Luis. 2016. «Buen o buena docente desde la perspectiva de estudiantes que han egresado de educación secundaria». Revista Educare Vol.20 No.3 Heredia Sep./Dec. 2016. <http://www.scielo.sa.cr/scielo.php?script=sci_arttext\&pid=S1409-42582016000300075> [Consulta 15-12-2016].

Centro interuniversitario de desarrollo CINDA, MINEDUC, 2007. Evaluación del desempeño docente y calidad de la docencia universitaria. En U. Malaspina, Evaluación del desempeño docente en la PUCP (págs. 337-340). Lima: CINDA.

Echeverría, Benito. 2002. «Gestión de la competencia de acción profesional». Revista de Investigación Educativa, 20(1), 7-43.

García, José. 2000. "Qué factores extraclase o s esgos afectan la evaluación docente en la educación superior». Revista Mexicana de Investigación Educativa, 5(10), 303-325.

Haskell, R. E. (1997). «Academic freedom, tenure, and student evaluation of faculty: Galloping polls in the 21st century». Educational Policy Analysis Archives, núm. 5 (6) revista electrónica. <http: //www.redalyc.org/ pdf/140/14001006.pdf> [Consulta: 14-12-2016] 
López, Carmen; Benedito, Vicente; y León, María. 2016. «El Enfoque de Competencias en la Formación Universitaria y su Impacto en la Evaluación». La Perspectiva de un Grupo de Profesionales Expertos en Pedagogía. Revista de Formación Universitaria Vol. 9(4), 11-22 (2016). <http://www.scielo.cl/pdf/formuniv/ v9n4/art03.pdf> [Consulta 25-09-2016].

Luna, Edna; Calderón, Nohemí; Caso Joaquín y Cordero, Graciela. 2012. «Diseño, desarrollo y validación de un cuestionario de evaluación de la competencia docente por los estudiantes». Revista de evaluación educativa, 1-22. <http://revalue.mx/ revista/index.php/revalue/issue/current> [Consulta 10-04-2016].

Mas, Oscar. 2012. Las competencias del docente universitario: la percepción del alumno, de los expertos y del propio protagonista. REDU - Revista de Docencia Universitaria, 10 (2), pp. 299-318.

Odar, América. 2007. Evaluación de la actividad docente de los profesores universitarios por los alumnos. Santiago: Fondo interuniversitario MINEDUC Chile.

Smith, R. A. y Cranton, P. A. (1992). «Students’ perceptions of teaching skills and overall effectiveness across instructional settings». Research in Higher Education (33), pp. 747-764.

Villa, Aurelio y Villa, Olga. 2007. El Aprendizaje basado en competencias y el desarrollo de la dimensión social en la universidad. Lima: Educar.

Presentado: $24 / 01 / 2017$

Aceptado: 26/04/2017

\section{Correspondencia}

Guillermo Napoleón Peláez Díaz

emperador0604@hotmail.com 